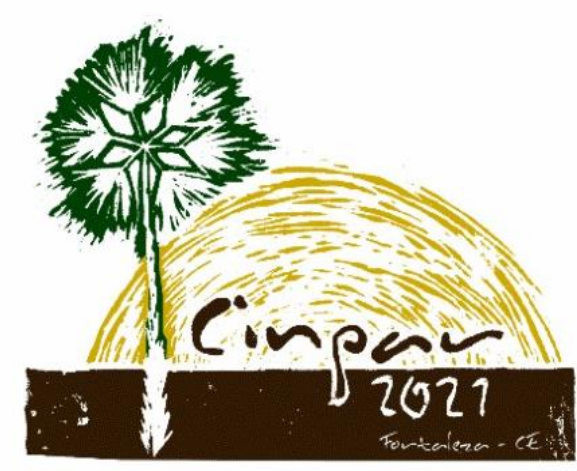

XVII Congresso Internacional sobre Patologia e Reabilitação das Construções

XVII Congreso Internacional sobre Patología y Rehabilitación de las Construcciones

XVII International Conference on Pathology and Constructions Rehabilitation

FORTALEZA (Brasil), 3 a 5 de junho de 2021

https://doi.org/10.4322/CINPAR.2021.125

\title{
Desempenho Mecânico do Concreto com Misturas Binárias e Ternárias de Cimento Portland, Sílica Ativa e Nanossílica
}

\section{Mechanical Performance of Concrete with Binary and Ternary Mixtures of Portland Cement, Silica Fume and Nanosilica}

\author{
João Paulo Fernandes dos SANTOS ${ }^{1}$, Ivan Dias Marques MACEDO ${ }^{2}$, Yuri Sotero Bomfim FRAGA ${ }^{3}$ \\ ${ }^{1}$ Centro Universitário Euro-Americano (UNIEURO), Brasília, Brasil, joaopaulofs31@gmail.com \\ ${ }^{2}$ Centro Universitário Euro-Americano (UNIEURO), Brasília, Brasil, ivanmacedo697@gmail.com \\ ${ }^{3}$ Centro Universitário Euro-Americano (UNIEURO), Brasília, Brasil, yurisotero.engcivil@gmail.com
}

\begin{abstract}
Resumo: Com a evolução da construção civil, aumentou-se também a necessidade de concretos com maiores resistências, incentivando o estudo de novos materiais que possam melhorar o desempenho do concreto. Desta forma, esta pesquisa tem como objetivo analisar a influência da utilização de sílica ativa (SA) e nanossílica (NS) no desempenho mecânico de concretos. Foram estudados quatro concretos, sendo um referência com $100 \%$ cimento Portland CP V-ARI, um com adição de $10 \%$ de SA, um com adição de $2 \%$ de NS e um com adição conjunta de $8 \%$ de SA e $2 \%$ de NS, sendo todas as adições em relação à massa de cimento. No estado fresco foi avaliada como a incorporação desses materiais influenciou na consistência dos concretos. No estado endurecido, foi realizado o ensaio de resistência à compressão dos concretos aos 1, 3, 7 e 21 dias de hidratação. Verificou-se que a adição de SA e/ou NS reduziu a fluidez dos concretos em relação ao concreto referência. No estado endurecido, observou-se aumento no desempenho mecânico do concreto com adição de SA, porém a adição de NS não resultou em diferenças significativas comparado com o concreto referência. Dessa forma, foi possível concluir que a utilização de SA foi mais eficaz para o aumento das propriedades mecânicas do concreto do que a utilização de NS.
\end{abstract}

Palavras-chave: Concreto, Nanossílica, Resistência à compressão, Sílica ativa.

Abstract: With the evolution of civil construction, it was increased also the need for concrete with higher strengths, encouraging the study of new materials that can improve concrete performance. Thus, this research aims to analyze the influence of the use of silica fume (SF) and nanosilica (NS) on the mechanical performance of concrete. Four concretes were studied, one reference with $100 \%$ Portland cement CP V-ARI, one with the addition of $10 \%$ of SF, one with the addition of $2 \%$ of NS and one with a combined addition of $8 \%$ of SF and $2 \%$ of NS, with all additions in relation to the cement weight. In the fresh state it was evaluated how the incorporation of these materials influenced the consistency of the concretes. In the hardened state, the concrete compressive strength test was performed at 1, 3, 7 and 21 days of hydration. It was found that the addition of SF and/or NS reduced the fluidity of the concretes in relation to the reference concrete. In the hardened state, there was an increase in the mechanical performance of the concrete with the addition of SF, however the addition of NS did not result in significant differences compared to the reference concrete. Thus, it was possible to conclude that the use of SF was more effective for increasing the mechanical properties of concrete than the use of NS.

Keywords: Concrete, Nanosilica, Compressive strength, Silica fume. 


\section{INTRODUÇÃO}

A construção civil vem evoluindo, cada vez mais, ao longo da história. Grande parte dessa evolução é consequência dos diversos estudos relacionados ao comportamento dos materiais utilizados nas edificações. O concreto, por exemplo, é o material mais utilizado nas construções e com isso ganha uma grande notoriedade nas pesquisas atuais (MEHTA; MONTEIRO, 2014).

A resistência mecânica é uma das principais características do concreto. É notória a necessidade de desenvolvimento de concretos com maior desempenho mecânico, conhecido como concreto de alto desempenho (CAD), para atender à demanda de estruturas com maior índice de esbeltez e com maiores vãos. Para isso, são realizados estudos que utilizam materiais cimentícios suplementares (MCSs) e nanomateriais que reagem com o hidróxido de cálcio $(\mathrm{CH})$ proveniente das reações de hidratação do cimento para formar silicato de cálcio hidratado (C-S-H) adicional, que é um dos responsáveis pelo aumento da resistência à compressão do concreto, processo conhecido como reação pozolânica (ANDRADE et al., 2018; FRAGA et al., 2020a).

Dentre os MCSs altamente reativos mais investigados para a produção do CAD destaca-se a sílica ativa (SA), enquanto como nanomaterial a nanossílica (NS) é bastante estudada. A SA é um material amorfo decorrente do processo de fabricação do silício metálico ou ferro silício através de processos metalúrgicos, apresentando dimensões micrométricas, tendo cerca de $94 \%$ da sua composição de SiO2. Este material contribui para o consumo de $\mathrm{CH}$ e formação de $\mathrm{C}-\mathrm{S}-\mathrm{H}$ adicional, bem como para o refinamento da estrutura porosa de compósitos cimentícios, resultando, consequentemente, no aumento do desempenho mecânico desses materiais (HERMANN et al., 2016; ROMANO et al., 2008; VASKE; CAMPAGNOLO; DAL MOLIN, 2010).

A nanossílica também é um material amorfo, que é composto por materiais granulares com tamanhos na escala nanométrica, também possuindo teor de $\mathrm{SiO}_{2}$ superior a $90 \%$. Assim como a $\mathrm{SA}$, este material proporciona formação de C-S-H adicional e refinamento da estrutura porosa dos compósitos cimentícios. A NS difere da SA não apenas pela sua escala de tamanho, como também pelo seu efeito em pastas, argamassas e concretos. Em geral, a NS proporciona aumento no desempenho mecânico a partir de 1 dia de hidratação devido à sua elevada reatividade, enquanto na SA esse aumento no desempenho mecânico é mais lento, geralmente a partir dos 3 dias, sendo observados efeitos também em idades posteriores como 28 dias de hidratação (ANDRADE et al., 2018; CAl et al., 2017; FRAGA et al., 2020b).

Esses materiais podem ser utilizados isoladamente ou de maneira simultânea em compósitos cimentícios. Na pesquisa realizada por Fraga et al. (2020b) foram estudadas pastas binárias e ternárias de cimento Portland, sílica ativa e nanossílica. Foram investigados os teores de $10 \%$ de sílica ativa, $2 \%$ de nanossílica e $8 \%$ de sílica ativa em conjunto com $2 \%$ de nanossílica. Os autores relataram um efeito sinérgico quando a sílica ativa foi utilizada em conjunto com a nanossílica, resultando no refinamento da estrutura porosa e em maior desempenho mecânico em comparação com as misturas binárias aos 28 dias de hidratação.

Diante do exposto, esta pesquisa teve como objetivo analisar o efeito incorporação de sílica ativa e nanossílica em misturas binárias e ternárias na consistência e no desempenho mecânico do concreto aos 1 , 3, 7 e 21 dias de hidratação.

\section{METODOLOGIA}

\subsection{Preparação dos Concretos}

Para a realização desta pesquisa foram utilizados cimento Portland CP V-ARI, SA, NS, agregado miúdo, agregado graúdo e aditivo superplastificante à base de policarboxilato. Foram estudados quatro diferentes concretos, sendo um referência e outros três com adição de $10 \%$ de adição de sílica ativa (10SA), $2 \%$ de nanossílica (2NS) e adição conjunta de $8 \%$ de SA e $2 \%$ de NS (8SA2NS). Estes teores foram escolhidos com base na literatura (ANDRADE et al., 2019; FRAGA et al., 2020a; FRAGA et al., 2020b). A nomenclatura e composição dos concretos estão descritos no Quadro 1. 
Quadro 1 - Nomenclatura e composição dos concretos

\begin{tabular}{|c|l|}
\hline Concreto & \multicolumn{1}{|c|}{ Composição } \\
\hline REF & Concreto com 100\% CP V-ARI \\
\hline $10 S A$ & Concreto com 100\% CP V-ARI + 10\% de sílica ativa \\
\hline 2 NS & Concreto com 100\% CP V-ARI + 2\% de nanossílica \\
\hline 8SA2NS & Concreto com 100\% CP V-ARI + 2\% de nanossílica + 8\% de sílica ativa \\
\hline
\end{tabular}

O traço de referência (REF) utilizado foi de 1:1,57:1,93 com relação água/cimento de 0,45 e teor de aditivo superplastificante de 0,4\%, obtido a partir da metodologia de dosagem descrita por Helene e Terzian (1992). A partir do traço do concreto REF foram preparados os outros concretos, mantendo o traço e a relação água/cimento, variando apenas o teor de aditivo superplastificante para obter o abatimento de $100 \pm 20 \mathrm{~mm}$ no ensaio de slump test de acordo com a NBR NM 67 (ABNT, 1998) e acrescentando SA e/ou NS.

Foram produzidos 48 corpos de prova cilíndricos com $100 \mathrm{~mm}$ de diâmetro e $200 \mathrm{~mm}$ de altura. Após a moldagem, os corpos de prova foram cobertos com uma lona plástica durante 24 horas para evitar a perda de água do concreto por evaporação. Em seguida, os corpos de prova foram desmoldados e colocados totalmente submersos em água saturada de cal até a realização dos ensaios para verificação do desempenho mecânico.

\subsection{Ensaio de Resistência a Compressão}

O ensaio de resistência a compressão em corpos de prova cilíndricos é normatizado pela NBR 5739 (ABNT, 2018). Para esse ensaio, foram utilizados três corpos de prova cilíndricos para cada traço por idade avaliada, sendo estes preparados conforme a NBR 5738 (ABNT, 2018). Os corpos de prova foram rompidos para verificação da resistência à compressão aos 1, 3, 7 e 21 dias de hidratação, obedecendo o tempo de tolerância para ruptura estabelecido pela NBR 5739 (ABNT, 2018). Como o cimento utilizado foi de alta resistência inicial, a resistência à compressão aos 21 dias é equivalente aos valores que seriam encontrados aos 28 dias de hidratação. Além disso, a NS possui maior efeito nos compósitos cimentícios até os 3 dias de hidratação e a SA promove a reação pozolânica a partir dos 7 dias de hidratação (FRAGA et al., 2020b; HARUEHANSAPONG et al., 2014; KHALOO et al., 2016; LI et al., 2017). Dessa forma, o efeito desses dois materiais pode ser observado nas idades mencionadas anteriormente.

Após a obtenção da resistência à compressão dos corpos de prova, foi realizada uma análise de variância ANOVA considerando nível de significância 0,05 , tendo-se como objetivo verificar se as adições de SA, NS influenciam de forma significativa na resistência à compressão do concreto. Constatada influência significativa, foi realizado o teste de Duncan com auxílio do software Statistica v.10, com intervalo de confiança de $95 \%$. O objetivo deste teste foi classificar os traços de concreto em grupos homogêneos e heterogêneos de resistência à compressão, levando em consideração a média e o desvio padrão.

\section{RESULTADOS}

\subsection{Teor de Aditivo Superplastificante}

Na Figura 1, estão apresentados os teores de aditivo superplastificante necessários para se obter o abatimento de $100 \pm 20 \mathrm{~mm}$ no ensaio de slump test. 


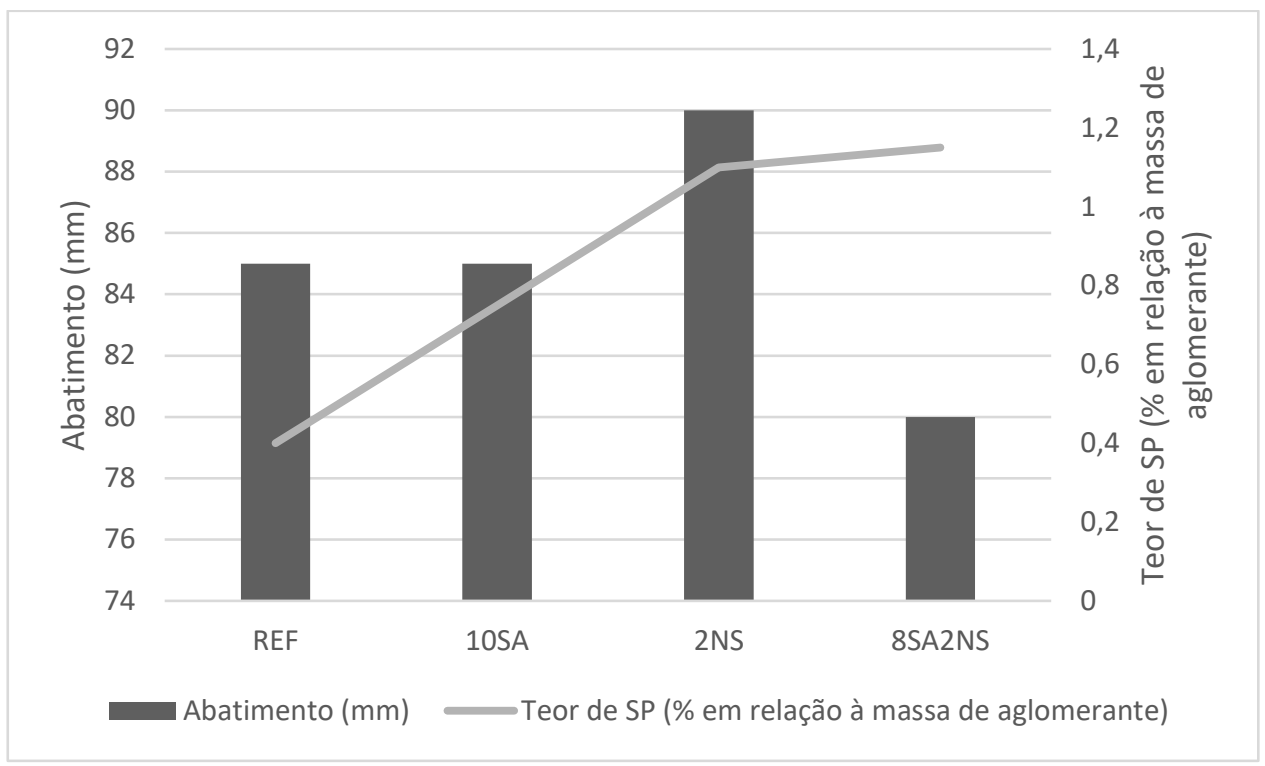

Figura 1- Teor de aditivo superplastificante necessário para se obter o abatimento de $100 \pm 20 \mathrm{~mm}$ dos concretos

Após realizada a determinação do teor de aditivo superplastificante pode-se observar que os traços contendo os SA e/ou NS necessitaram de um teor maior de aditivo SP quando comparado ao referência, pois na hora da realização do ensaio apresentaram uma consistência maior. Este fato foi explicado por Abd.El.Aleem et al. (2014), Mehta; Monteiro (2014) e Singh et al. (2012), que justificaram o aumento da demanda de água ou aditivo superplastificante devido à grande área superficial que materiais finos como SA e NS apresentam.

\subsection{Resistência à Compressão}

Os resultados obtidos com o ensaio resistência à compressão dos concretos aos 1, 3, 7 e 21 dias estão apresentados na Figura 2.

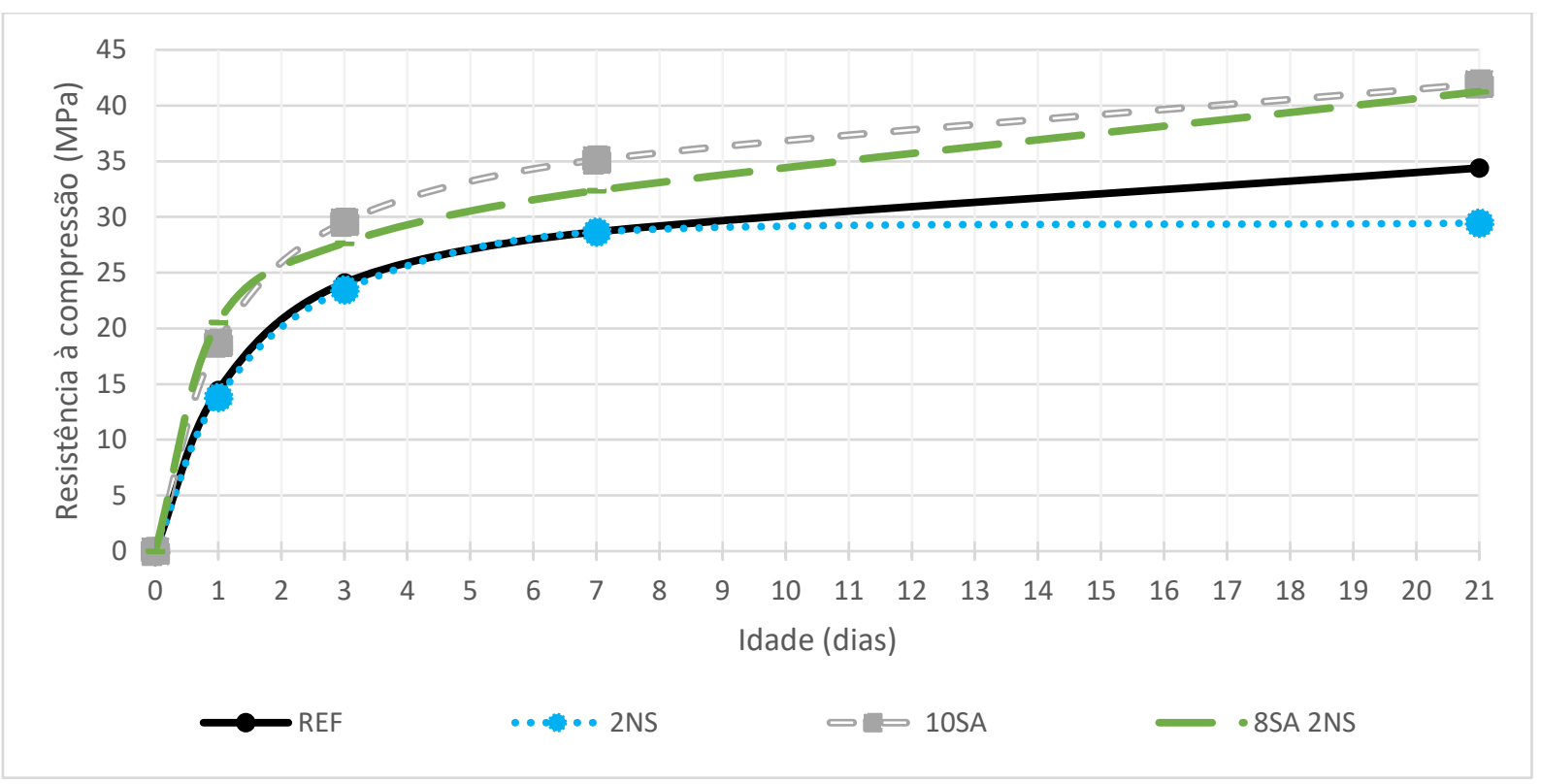

Figura 2- Resistência à compressão dos concretos aos 1, 3, 7 e 21 dias de hidratação

Com 1 dia de hidratação o concreto 8SA2NS obteve a maior resistência média à compressão, resultando no valor de 20,6 MPa, ou seja, resistência de um concreto estrutural. Nesta idade, os concretos 10SA, REF e 2NS resultaram em resistências médias à compressão de 18,7 MPa, 14,4 MPA e 13,8 MPa, respectivamente. Com 3 dias de hidratação todos os concretos apresentam ganhos de resistências, sendo o 10SA com a maior resistência (29,5 MPa) e o 2NS a menor com 23,5 MPa. Aos 7 dias os concretos continuaram tendo ganhos 
de resistência, sendo mais acentuado no 10SA com 35,1 MPa, seguido de 8SA2NS com 32,4 MPa, e os concreto REF e 2NS apresentaram a mesma resistência de 28,7 MPa. Na última idade o $2 \mathrm{NS}$ apresentou pouco ganho de resistência em ralação a idade anterior com 29,5 MPa, os demais apresentaram ganhos mais relevantes com o 10SA chegando a 42,1 MPa e o 8SA2NS a 41,3 MPa.

Como observado no ensaio de resistência à compressão, alguns concretos resultaram em desempenhos mecânicos semelhantes. Para verificar se as diferenças de resistência foram significativas, foi realizada a análise de variância one-way ANOVA, conforme mostrado no Quadro 2.

Quadro 2 - Análise de variância (one-way ANOVA) da resistência à compressão axial dos concretos aos 1, 3, 7 e 21 dias de hidratação

\begin{tabular}{|c|c|c|c|c|c|}
\hline Idade & SQ & $M Q$ & $\mathbf{F}$ & p-valor & Resultado \\
\hline $1 \mathrm{dia}$ & 96,4 & 32,1 & 23,1 & 0,000271 & Significativo \\
\hline 3 dias & 76,4 & 25,5 & 9,1 & 0,005967 & Significativo \\
\hline 7 dias & 90,4 & 30,1 & 13,0 & 0,001908 & Significativo \\
\hline 21 dias & 322,2 & 107,4 & 4,2 & 0,045695 & Significativo \\
\hline \multicolumn{6}{|c|}{$\begin{array}{l}\text { Sendo: } \\
\text { SQ= Soma dos quadrados; } \\
\text { MQ= Média dos quadrados; } \\
\text { F= Parâmetro de Fisher para o teste de significância; } \\
\text { p-valor= probabilidade de significância. }\end{array}$} \\
\hline
\end{tabular}

Na análise de variância ANOVA, apresentada no Quadro 2, foi constatado um p-valor menor que o nível de significância de 0,05 para todas as idades de hidratação. Dessa forma, foi constatado que houve diferença significativa em todas as idades. Para refinar a análise, foi realizado o teste de Duncan, para observar quais concretos apresentaram resistências homogêneas e heterogêneas, conforme apresentado no Quadro 3.

Quadro 3 - Resultados do teste de Duncan para resistência à compressão dos concretos aos 1,3, 7 e 21 dias de hidratação

\begin{tabular}{|c|c|c|c|c|c|c|}
\hline Idade & Concreto & $\begin{array}{l}\text { Resistência média à } \\
\text { compressão (MPa) }\end{array}$ & $\begin{array}{c}\text { Desvio padrão } \\
\text { (MPa) }\end{array}$ & Grupo 1 & Grupo 2 & Grupo 3 \\
\hline \multirow{4}{*}{$1 \mathrm{dia}$} & REF & 14,4 & 0,7 & $x$ & & \\
\hline & $2 \mathrm{NS}$ & 13,8 & 2,0 & $x$ & & \\
\hline & $10 \mathrm{SA}$ & 18,7 & 0,7 & & $x$ & \\
\hline & 8SF2NS & 20,6 & 0,1 & & $x$ & \\
\hline \multirow{4}{*}{3 dias } & REF & 24,1 & 1,1 & $x$ & & \\
\hline & $2 \mathrm{NS}$ & 23,5 & 0,4 & $x$ & & \\
\hline & $10 \mathrm{SA}$ & 29,5 & 2,8 & & $x$ & \\
\hline & 8SA 2NS & 27,7 & 1,4 & & $x$ & \\
\hline \multirow{4}{*}{7 dias } & REF & 28,7 & 1,4 & $x$ & & \\
\hline & $2 \mathrm{NS}$ & 28,7 & 0,2 & $x$ & & \\
\hline & $10 \mathrm{SA}$ & 35,1 & 0,1 & & & $x$ \\
\hline & 8SA2NS & 32,4 & 2,7 & & $x$ & \\
\hline \multirow{4}{*}{21 dias } & REF & 34,4 & 4,7 & $x$ & $x$ & \\
\hline & $2 N S$ & 29,5 & 2,0 & $x$ & & \\
\hline & $10 S A$ & 42,1 & 5,0 & & $x$ & \\
\hline & 8SA2NS & 41,3 & 7,1 & & $x$ & \\
\hline
\end{tabular}

Para as idades de 1, 3 e 21 dias de hidratação, foram formados apenas 2 grupos de resistência à compressão, sendo o grupo 1 de menor resistência e o grupo 2 de maior resistência. Aos 7 dias de hidratação foram 
formados 3 grupos, sendo o grupo 1 classificado como de menor resistência, o grupo 2 de resistência intermediária e o grupo 3 de maior resistência à compressão.

Ao analisar os resultados obtidos, foi observado que as resistências com um dia dos traços REF e 2NS foram classificas no grupo 1, ou seja, são iguais. Esse resutado diverge do que é apresentado na literatura, em que era esperado para o 2 NS altas resistências iniciais devido à sua alta reatividade pozolânica, como apresentado nos estudos de Noli, (2015); Qing et al. (2007). Nesta idade, os traços 8SA2NS e 10SA foram classificados no grupo 2, ou seja, apresentaram maiores resistências inicias em relação ao traço referência, confirmando o que já é visto na literatura, que justifica esse aumento no desempenho mecânico com 1 dia ao efeito fíler, no qual grãos menores de sílica ativa preenchem os vazios entre os grãos maiores de cimento Portland (HERMANN et al. ,2016; MEDEIROS et al., 2017; SOUSA, 2017; FRAGA et al., 2020b).

Aos três dias de hidratação o traço 2NS continuou ganhando resistência de forma semelhante ao traço referência, permanecendo os mesmos no grupo 1 . Nesta idade, devido à formação de C-S-H adicional oriundo da reação pozolânica da NS com o $\mathrm{CH}$ proveniente da hidratação do cimento Portland, esperava-se que o concreto 2NS resultasse em maior desempenho mecânico que o concreto REF, o que não foi observado, sendo necessário estudos complementares para justificar esse comportamento que pode ser devido ao aumento da retração provocado pela NS. Os traços 10SA e 8SA2NS continuaram tendo ganho de resistência superiores ao traço REF e permanecendo no grupo 2, o que pode ser justificado pela ação combinada do efeito fíler com a reação pozolânica da SA com o CH (NOLI, 2015; QING et al., 2007; FRAGA et al., 2020b).

Aos sete dias de hidratação, observou-se que os traços REF e 2NS resultaram em resistências semelhantes entre si e menores do que os traços 10SA e 8SA2NS. Ao comparar os resultados dos traços 10SA e 8SA2NS, observou-se que o concreto 10SA resultou em maior resistência à compressão em relação ao traço 8SA2NS. Esse comportamento não era esperado, tendo em vista que a literatura aborda um efeito sinérgico ao utilizar a SA em conjunto com a NS, resultando na formação de um maior teor de C-S-H, redução da porosidade e maior refinamento da estrutura porosa em comparação com a utilização apenas da SA (ANDRADE et al., 2019; FRAGA et al., 2020b; GHAFOORI et al., 2018; GHODDOUSI et al., 2018; Ll et al., 2017).

Assim como nas demais idades, aos 21 dias de hidratação os concretos resultaram no mesmo comportamento. Os concretos REF e $2 \mathrm{NS}$ resultaram nas menores resistências à compressão, sendo classificados no grupo 1, enquanto os concretos 10SA e 8SA2NS resultaram nas maiores resistências, sendo classificados no grupo 2. Conforme descrito anteriormente, a adição de NS nos concretos divergiu dos resultados encontrados na literatura, sendo esperado grande aumento de resistência à compressão nas idades iniciais. Dentre as hipóteses para justificar esse resultado estão o efeito negativo que a NS causa na retração do concreto ou que o teor da NS utilizada não foi efetivo para promover a reação pozolânica (ARAÚJO; VIANA, 2020).

Dessa forma, a utilização de nanossílica não fez com que os traços 2NS e 8SA2NS, atingissem resultados semelhantes ao que é encontrado na literatura. Para o 2NS eram esperadas altas resistências iniciais e para o traço 8SA2NS tinha-se como objetivo ganho de resistência nas idades iniciais devido a presença de nanossílica e que a resistência continuasse aumentando ao longo do tempo por conta da presença da sílica ativa, que proporciona uma reação pozolânica mais lenta (FRAGA et al., 2020b).

\section{CONCLUSÕES}

Esta pesquisa objetivou avaliar a influência da adição de SA e NS em misturas binárias e ternárias com cimento Portland no estado fresco e no estado endurecido de concretos. No estado fresco, a adição de SA e/ou NS resultou na diminuição da fluidez do concreto, sendo necessário adicionar maior teor de aditivo superplastificante para os concretos apresentarem a mesma consistência que o concreto somente com cimento Portland.

No estado endurecido, a SA mostrou-se bastante eficaz no aumento do desempenho mecânico de concretos a partir de 1 dia de hidratação, devido ao efeito fíler, até 21 dias de hidratação, idade na qual esse efeito é atribuído principalmente à reação pozolânica. Esperava-se que a adição de NS resultasse no aumento do desempenho mecânico principalmente entre 1 e 3 dias, porém não houve diferença significativa da incorporação desse material no desempenho mecânico do concreto comparado com o concreto referência. 
Diante do exposto, pode-se concluir que para os resultados encontrados nesta pesquisa a adição de SA foi mais eficiente do que a utilização de NS para a produção de concretos com maiores resistências. Apesar disso, como os resultados da incorporação de NS não seguiram o mesmo padrão de aumento da resistência à compressão encontrado na literatura, recomenda-se que em pesquisas futuras sejam realizadas novas investigações sobre o desempenho desse material no concreto, como por exemplo:

- Investigar o efeito de diferentes teores de NS no desempenho mecânico do concreto;

- Analisar a influência da NS na retração do concreto;

- Avaliar o desempenho mecânico de concretos com diferentes tipos de NS;

- Verificar o efeito da incorporação de SA e NS em misturas binárias e ternárias quanto à durabilidade do concreto.

\section{REFERÊNCIAS BIBLIOGRÁFICAS}

Abd.El.Aleem, S.; Heikal, M.; Morsi, W. M. (2014). Hydration characteristic, thermal expansion and microstructure of cement containing nano-silica. Construction and Building Materials, v. 59, p. 151-160.

ABNT, Associação Brasileira de Normas Técnicas. (1998). NBR NM 67: Concreto- Determinação da consistencia pelo abatimento do tronco de cone - Requisitos. Rio de Janeiro.

ABNT, Associação Brasileira de Normas Técnicas. (2015). NBR 5738: Concreto - Procedimento para moldagem e cura de corpos de prova. Rio de Janeiro.

ABNT, Associação Brasileira de Normas Técnicas. (2018). NBR 5739: Concreto - ensaio de compressão de corpos de prova cilíndricos. Rio de Janeiro.

Andrade, D.S.; Rêgo, J. H.S.; Morais, P.C.; Rojas, M. F. (2018). Chemical and mechanical characterization of ternary cement pastes containing metakaolin and nanosilica. Construction and Building Materials, v. 159, p. 18-26.

Araújo, R. L. R.; Viana, K. H. L. (2020). Efeito do teor de nanossílica no desempenho mecânico do microconcreto. 2020. 20 f. Trabalho de conclusão de curso (Graduação em Engenharia Civil) - Centro Universitário Euro-Americano, Brasília.

Cai, Y.; Hou, P.; Cheng, X.; Du, P.; Ye, Z. (2017). The effects of nanoSiO2 on the properties of fresh and hardened cement-based materials through its dispersion with silica fume. Construction and Building Materials, v. 148, p. 770-780.

Fraga, Y. S. B.; Rêgo, J. H. S.; Capuzzo, V. M. S.; Andrade, D. S. (2020a). Efeito da ultrasonicação da sílica ativa e da nanossílica coloidal em pastas de cimento. Revista Matéria, v. 25, n.4, p. 1-16.

Fraga, Y. S. B.; Rêgo, J. H. S.; Capuzzo, V. M. S.; Andrade, D. S.; Morais, P. C. (2020b). Ultrasonication and synergistic effects of silica fume and colloidal nanosilica on the C-S-H microstructure. Journal of Building Engineering, v. 32, p. 1-13.

Ghafoori, N.; Batilov, I.; Najimi, M. (2017). Influence of dispersion methods on sulfate resistance of nanosilicacontained mortars. Journal of Materials in Civil Engineering, v. 29, p. 1-13.

Ghoddousi, P.; Javid, A. A. S.; Zareechian, M.; Korayem, A. H. (2018). Physical and chemical effects of siliceous particles at nano, micro, and macro scales on properties of self-consolidating mortar overlays. Construction and Building Materials, v. 189, p. 1140-1154.

Haruehansapong, S.; Pulngern, T.; Chucheepsakul, S. (2014). Effect of the particle size of nanosilica on the compressive strength and the optimum replacement content of cement mortar containing nano-SiO2. Construction and Building Materials, v. 50, p. 471-477.

Helene, P., Terzian, P. (1992). Manual de dosagem e controle do concreto, São Paulo, PINI. SENAI.

Hermann, A.; Langaro, E. A.; Silva, S. H. L.; Klein, N. S. (2016). Empacotamento de partículas de cimento e sílica ativa em pastas pelo uso de modelo analítico. Revista Ibracon de Estruturas e Materiais, v. 9, n. 1, p. 48-65.

Khaloo, A.; Mobini, M. H.; Hosseini, P. (2016). Influence of different types of nano-SiO2 particles on properties of high-performance concrete. Construction and Building Materials, v. 113, p. 188-201. 
Li, L. G.; Huang, Z. H.; Zhu, J.; Kwan, A. K. H.; Chen, H. Y. (2017). Synergistic effects of micro-silica and nanosilica on strength and microstructure of mortar. Construction and Building Materials, v. 140, p. 229-238.

Medeiros, M. H. F.; Raisdorfer, J.; Filho, J. H. (2017). Influência da sílica ativa e do metacaulim na velocidade de carbonatação do concreto: relação com resistência, absorção e relação a/c. Ambiente Construído, v. 17 , n. outubro, p. 125-139.

Mehta, P.K.; Monteiro, P.J.M. (2014). Concreto: Microestrutura, Propriedades e Materiais. 2a Edição, São Paulo, IBRACON.

Noli, P. R. F. O. (2015). Propriedades mecânicas do concreto de alto desempenho com adição de nanosílica estabilizada. Tese (Mestrado em Estruturas e Construção Civil) - Universidade Federal de Minas Gerais, Minas Gerais.

Qing, Y.; Zenan, Z.; Deyu, K.; Rongshen, C. (2007). Influence of nano-SiO2 addition on properties of hardened cement paste as compared with silica fume. Construction and Building Materials, v. 21, p. 539-545.

Romano, R. C. O.; Schreurs, H.; John, V. M.; Pileggi, R. G. (2008). Influência da técnica de dispersão nas propriedades da sílica ativa. Cerâmica, v. 54, p. 456-461.

Singh, L. P.; Bhattacharyya, S. K.; Ahalawat, S. (2012). Preparation of size controlled silica nano particles and its functional role in cementitious system. Journal of Advanced Concrete Technology, v. 10, p. 345-352.

Sousa, I. P. S. (2017). Obtenção de nanossílica funcionalizada visando o uso como aditivo em misturas cimentícias. 72 f. Dissertação (Mestrado em Química) - Universidade Federal de Goiás, Goiânia.

Vaske, N. R.; Campagnolo, J. L.; Dal Molin, D. C. C. (2010). Relationship between the compressive strength of silica fume mortar applied to the substratum and the one obtained in standardized cylindrical test specimens. Revista Ibracon de Estruturas e Materiais, v. 3, n. 3, p. 322-345. 